\title{
A Generalization of Rennie's Inequality
}

\author{
A. J. Goldman
}

(February 25, 1964)

\begin{abstract}
A generalization is given of Rennie's recent extension of the Kantorovich inequality. The result is used in an alternate proof of the sharp upper bounds for ratios of weighted means obtained by Cargo and Shisha.
\end{abstract}

\section{Introduction}

For $n>1$, consider a sequence of $2 n$ positive numbers

$$
x_{1}, \ldots, x_{n} ; w_{1}, \ldots, w_{n}
$$

not necessarily distinct, and such that

$$
0<m \leqslant x_{i} \leqslant M ; w_{1}+\ldots+w_{n}=1
$$

where $M$ and $m$ are constants. A well-known and useful inequality due to Kantorovich [3] $]^{2}$ asserts that under these circumstances

$$
\left(\sum w_{i} x_{i}\right) \cdot\left(\sum w_{i} x_{i}^{-1}\right) \leqslant(M+m)^{2} / 4 M m
$$

Recently Rennie [4] gave a simple proof of an inequality which in turn yields an especially elegant derivation of (1). Also recently, Cargo and Shisha [1] obtained a general inequality which gives sharp upper bounds for the ratios of weighted means,

$$
\left(\sum w_{i} x_{i}^{s}\right)^{1 / s}\left(\sum w_{i} x_{i}^{r}\right)^{-1 / r} \leqslant\left\{r^{-1}\left(q^{r}-1\right)\right\}^{-1 / s}\left\{s^{-1}\left(q^{s-1}\right)\right\}^{1 / r}\left\{(s-r)^{-1}\left(q^{s}-q^{r}\right)\right\}^{(1 / s)-(1 / r)}
$$

where $q=M / m$, valid for real $s$ and $r$ satisfying ${ }^{3}$

$$
s>r, s r \neq 0 .
$$

The inequality (1) is obtained by setting $s=1$ and $r=(-1)$.

Our purpose here is to see how Rennie's proof of (1) might be adapted to a proof of (2). Although the generalization is not quite as elegant as the original special case (the sources of the extra complexity are noted at appropriate points in the text), the resulting alternate proof of (2) and generalization of Rennie's inequality seem worth recording.

The notation employed will be appropriate to the continuous version of the results (which specializes to the discrete version); the "weights" $w_{i}$ of (1) and (2) are replaced by a fixed measure space of unit total measure, the numbers $x_{i}$ are replaced by a real-valued measurable function $x$ satisfying $0<m \leqslant x \leqslant M$ almost everywhere, and sums like $\Sigma w_{i} x_{i}$ or $\Sigma w_{i} x^{s}$ are replaced by integrals

\footnotetext{
${ }^{1}$ The author is indebted to K. Goldberg (NBS Numerical Analysis Section) for helpful discussions.

${ }^{2}$ Figures in brackets indicate the literature references at the end of this paper.

${ }^{3}$ The limiting cases with $s r=0$ are also treated in [1], but will be omitted here.
} 
$I(x)$ or $I\left(x^{s}\right)$ taken over the entire space. It will be convenient to normalize by replacing $x$ with the measurable function $y=x / m$, which satisfies

$$
1 \leqslant y \leqslant q=M / m
$$

almost everywhere. Our generalization of Rennie's inequality (which after "denormalization" is the special case $s=1, r=(-1))$ asserts under hypotheses (3) that

$$
\begin{array}{ll}
q^{s}-q^{r} \leqslant\left(q^{s}-1\right) I\left(y^{r}\right)-\left(q^{r}-1\right) I\left(y^{s}\right) & \text { if } s r>0, \\
q^{s}-q^{r} \geqslant\left(q^{s}-1\right) I\left(y^{r}\right)-\left(q^{r}-1\right) I\left(y^{s}\right) & \text { if } s r<0 .
\end{array}
$$

For real $r \neq 0$, the $r$ th order mean of $x$ (with weights given by the measure) is defined as

$$
M_{r}(x)=\left\{I\left(x^{r}\right)\right\}^{1 / r}
$$

In terms of these means we can define the quotients

$$
Q_{s r}(x)=M_{s}(x) / M_{r}(x) .
$$

Since the quotients are unchanged by normalization, the continuous version of the Cargo-Shisha result (2) can be written

$$
Q_{s r}(y) \leqslant\left\{r^{-1}\left(q^{r}-1\right)\right\}^{-1 / s}\left\{s^{-1}\left(q^{s}-1\right)\right\}^{1 / r}\left\{(s-r)^{-1}\left(q^{s}-q^{r}\right)\right\}^{(1 / s)-(1 / r)}
$$

This and (4) will be proved in section 3, after disposing of some preliminaries in the following section 2 .

Two natural directions for possible further investigations suggest themselves. The first is to seek a generalized inequality involving more than two powers of $y$. The second is to consider integrals $I\{f(y)\}$ for functions $f$ other than power functions.

\section{Preliminaries}

The basis of the analysis turns out to be the famous inequality between the arithmetic and geometric means ${ }^{4}$, which asserts for $a>0$ and $b>0$ that

$$
\begin{array}{cc}
a^{\alpha} b^{1-\alpha} \leqslant \alpha a+(1-\alpha) b & \text { if } 0<\alpha<1, \\
a^{\alpha} b^{1-\alpha} \geqslant \alpha a+(1-\alpha) b & \text { if } \alpha<0,
\end{array}
$$

with equality if and only if $a=b$. The form $(6 \mathrm{~b})$ is obtained from the more familiar form (6a) by applying the latter to

$$
a^{\prime}=a^{1-\alpha}, b^{\prime}=b^{1-\alpha}, \alpha^{\prime}=-\alpha /(1-\alpha)
$$

and then multiplying the result through by $(1-\alpha) a^{\alpha}$ and rearranging terms. Rennie [4] required only the special case $\alpha=1 / 2$ which is particularly easy to prove ${ }^{5}$, but it is known [2] that the general case of (6a) can be deduced from this special case by elementary algebraic methods plus a simple limiting process to handle irrational $\alpha$.

As a first application of (6), we introduce the function ${ }^{6}$

$$
\varphi(u)=\varphi(u ; t, \tau)=\left(u^{\tau+t}-1\right) /\left(u^{t}-1\right) \quad(t \tau>0)
$$

and prove it to be strictly increasing for $u>0$ if $t>0$ and $\tau>0$, but strictly decreasing for $u>0$

${ }^{4}$ Cargo and Shisha [1] show how their inequality yields another proof of this classical one.

${ }^{5}$ Expand the left-hand side of $\left(a^{1 / 2}-b^{1 / 2}\right)^{2} \geqslant 0$ and rearrange terms.

${ }^{6}$ We give $\varphi(u)$ its limiting value $(\tau+t) / t$ at $u=1$. 
if $t<0$ and $\tau<0$. For this purpose, calculate for $u \neq 1$

$$
\varphi^{\prime}(u)=\left(u^{t}-1\right)^{2} u^{t-1}(\tau+t)\left\{\tau(\tau+t)^{-1} u^{\tau+t}+t(\tau+t)^{-1}-u^{\tau}\right\} .
$$

To prove that the last factor is positive, so that (as desired) the derivative has the same sign as $\tau+t$, we need only apply (6a) with

$$
a=u^{\tau+t}, b=1, \alpha=\tau(\tau+t)^{-1} .
$$

Rennie [4] required only the obvious special case that

$$
\varphi(u ; 1,1)=u+1
$$

is increasing.

Assuming (3) as always, we now show for $1 \leqslant Y \leqslant q$ that

$$
\begin{array}{ll}
\left(q^{s}-1\right) Y^{r}-\left(q^{r}-1\right) Y^{s} \geqslant q^{s}-q^{r} & \text { if } s r>0, \\
\left(q^{s}-1\right) Y^{r}-\left(q^{r}-1\right) Y^{s} \leqslant q^{s}-q^{r} & \text { if } s r<0,
\end{array}
$$

with equality if and only if $Y=1$ or $Y=q$. Clearly equality holds in the indicated cases, so we assume $1<Y<q$ and prove strict inequality. For $s r>0$, assertion (7a) is equivalent if $s>r>0$ to

$$
\left(q^{s}-1\right) /\left(q^{r}-1\right)=\varphi(q ; r, s-r)>\varphi(Y ; r, s-r)=\left(Y^{s}-1\right) /\left(Y^{r}-1\right),
$$

and if $r<s<0$ to

$$
\left(q^{r}-1\right) /\left(q^{s}-1\right)=\varphi(q ; s, r-s)<\varphi(Y ; s, r-s)=\left(Y^{r}-1\right) /\left(Y^{s}-1\right) .
$$

For $s r<0$, assertion ( $7 \mathrm{~b})$ is equivalent to

and thus to

$$
\left(Y^{s}-1\right) /\left(Y^{r}-1\right)<\left(q^{s}-1\right) /\left(q^{r}-1\right)
$$

$$
\left(Y^{s-r}-Y^{-r}\right) /\left(Y^{-r}-1\right)=\varphi(Y ;-r, s)-1<\varphi(q ;-r, s)-1=\left(q^{s-r}-q^{-r}\right) /\left(q^{-r}-1\right) .
$$

So (7) follows from the properties of $\varphi(u)$ proved in the last paragraph.

\section{Proofs of Results}

The values $Y$ of the normalized function $y$ obey $1 \leqslant Y \leqslant q$ almost everywhere, so that our generalized Rennie inequality (4) is obtained from (7) simply by integrating over the measure space. From the discussion of (7) it follows that equality occurs in (4) if and only if $y$ differs from 1 and $q$ only on a set of measure zero. 'Denote the measure by $\mu$, the inverse transformation of $y$ by $y^{-1}$ (to be distinguished from the function $1 / y$ obtained from $y^{r}$ for $r=(-1)$ ), and define

$$
\theta=\mu\left\{y^{-1}(q)\right\}
$$

then the condition for equality in (4) can be written

$$
1-\theta=\mu\left\{y^{-1}(1)\right\}
$$

For $s r>0$ rewrite (4a) as

$$
\left\{-r(s-r)^{-1}\right\}\left\{r^{-1}\left(q^{r}-1\right) I\left(y^{s}\right)\right\}+\left\{s(s-r)^{-1}\right\}\left\{s^{-1}\left(q^{s}-1\right) I\left(y^{r}\right)\right\} \geqslant(s-r)^{-1}\left(q^{s}-q^{r}\right),
$$


and for $s r<0$ rewrite $(4 \mathrm{~b})$ as

$$
\left\{-r(s-r)^{-1}\right\}\left\{r^{-1}\left(q^{r}-1\right) I\left(y^{s}\right)\right\}+\left\{s(s-r)^{-1}\right\}\left\{s^{-1}\left(q^{s}-1\right) I\left(y^{r}\right)\right\} \leqslant(s-r)^{-1}\left(q^{s}-q^{r}\right) .
$$

We shall apply the arithmetic-mean geometric-mean inequality (6), with ${ }^{7}$

$$
a=r^{-1}\left(q^{r}-1\right) I\left(y^{s}\right), b=s^{-1}\left(q^{s}-1\right) I\left(y^{r}\right), \alpha=-r(s-r)^{-1},
$$

to the left-hand side of (10). If $s>r>0$ we apply (6b) to (10a) and obtain

$$
\left\{r^{-1}\left(q^{r}-1\right) I\left(y^{s}\right)\right\}^{-r /(s-r)}\left\{s^{-1}\left(q^{s}-1\right) I\left(y^{r}\right)\right\}^{s /(s-r)} \geqslant(s-r)^{-1}\left(q^{s}-q^{r}\right),
$$

which when raised to the negative power $(s-r) /(-s r)$ yields

$$
\left\{r^{-1}\left(q^{r-1}\right) I\left(y^{s}\right)\right\}^{1 / s}\left\{s^{-1}\left(q^{s}-1\right) I\left(y^{r}\right)\right\}^{-1 / r} \leqslant\left\{(s-r)^{-1}\left(q^{s}-q^{r}\right)\right\}^{(1 / s)-(1 / r)} .
$$

If $s r<0$ we apply (6a) to (10b) to obtain the reverse of (12), which when raised to the positive power $(s-r) /(-s r)$ yields (13) again. Finally, if $r<s<0$ we shift from (11) to

$$
a=s^{-1}\left(q^{s-1)} I\left(y^{r}\right), b=r^{-1}\left(q^{r}-1\right) I\left(y^{s}\right), \alpha=s(s-r)^{-1}\right.
$$

and apply (6b) to (10a) to obtain (12), which when raised to the negative power $(s-r) /(-s r)$ yields (13). So in all cases we have (13), a rearrangement of the Cargo-Shisha inequality (5).

Equality can occur in (5) if and only if it occurs in (4), so that (9) holds, and also $a=b$ in (11) and (14). Substitution of (9) into this last condition leads to

$$
r^{-1}\left(q^{r}-1\right)\left\{\theta q^{s}+(1-\theta) \cdot 1\right\}=s^{-1}\left(q^{s}-1\right)\left\{\theta q^{r}+(1-\theta) \cdot 1\right\}
$$

or equivalently

$$
\theta=\theta(q ; s, r)=(s-r)^{-1}\left\{r\left(q^{r}-1\right)^{-1}-s\left(q^{s-1}\right)^{-1}\right\},
$$

the same condition for equality obtained in [1]. To show that the bounds in (5) are sharp, it suffices to prove that

$$
0<\theta(q ; s, r)<1 .
$$

The left-hand inequality is equivalent for $s>r>0$ to

$$
\left(q^{s}-1\right) /\left(q^{r}-1\right)=\varphi(q ; r, s-r)>\varphi(1 ; r, s-r)=s / r,
$$

for $r<s<0$ to

$$
\left(q^{r}-1\right) /\left(q^{s}-1\right)=\varphi(q ; s, r-s)<\varphi(1 ; s, r-s)=r / s,
$$

and for $s r<0$ to

$$
\left(q^{s-r}-q^{-r}\right) /\left(q^{-r}-1\right)=\varphi(q ;-r, s)-1>\varphi(1 ;-r, s)-1=s /(-r) .
$$

The right-hand inequality in (16) can be rewritten

$$
r /\left(q^{-r}-1\right)>s /\left(q^{-s}-1\right) .
$$

This is equivalent for $s>r>0$ to

$$
\left(q^{-s}-1\right) /\left(q^{-r}-1\right)=\varphi(q ;-r, r-s)<\varphi(1 ;-r, r-s)=s / r
$$

\footnotetext{
${ }^{7}$ From now on we exclude the trivial case $q=1$ and assume $q>1$.
} 
for $r<s<0$ to

$$
\left(q^{-r}-1\right) /\left(q^{-s}-1\right)=\varphi(q ;-s, s-r)>\varphi(1 ;-s, s-r)=r / s,
$$

and for $s r<0$ to

$$
\left(q^{-s}-1\right) /\left(q^{-r}-1\right)=1-\varphi(q ; r,-s)>1-\varphi(1 ; r,-s)=s / r .
$$

Thus (16) is verified.

Added in proof: Rennie's inequality turns out to be equivalent to an apparently stronger one given by Diaz and Metcalf [5] in the same year, and our generalization is in turn equivalent to one announced previously by Diaz and Metcalf [6]. The proofs of these (non-trivial) equivalences will be presented in a forthcoming paper by Diaz, Metcalf, and the writer.

\section{References}

[1] G. T. Cargo and O. Shisha, Bounds on ratios of means, J. Res. NBS 66B (1962).

[2] G. H. Hardy, J. E. Littlewood, and G. Polya, Inequalities (2d ed.), Cambridge Univ. Press (1952).

[3] L. V. Kantorovich, Functional analysis and applied mathematics, Uspekhi Math. Nauk 3, 89 (1948).

[4] B. C. Rennie, An inequality which includes that of Kantorovich, Amer. Math. Monthly 70 (1963).

[5] J. B. Diaz and F. T. Metcalf, Stronger forms of a class of inequalities of G. Polya-G. Szego and L. V. Kantorovich, Bull. Amer. Math. Soc. 69, 415-418 (1963).

[6] J. B. Diaz and F. T. Metcalf, On an inequality complementary to Holder's inequality, Notices Amer. Math. Soc. 10, No. 5, 510 (Aug. 1963).

(Paper 68B2-117) 